Proc. Estonian Acad. Sci. Eng., 2004, 10, 2, 137-147

\title{
Estimation of the finger arterial pressure-volume relationship and blood pressure waveform from photoplethysmographic signals
}

\author{
Jaak Talts \\ Department of Physiology, University of Tartu, Ülikooli 18, 51014 Tartu, Estonia; talts@ut.ee \\ Received 23 December 2003, in revised form 16 February 2004
}

\begin{abstract}
A method for deriving the finger arterial pressure-volume $(P-V)$ relationship and blood pressure waveform from two photoplethysmographic (volumetric) signals is presented. The new approach makes it possible to estimate also the beat-to-beat as well as instantaneous values of the dynamic arterial compliance of small arteries. The identification of the $P-V$ relationship was performed applying an asymmetric tangent approximation and non-linear fitting. The results of modelling demonstrate that two finger photoplethysmographic waveforms, recorded at different cuff pressure levels, can be used for the estimation of the finger arterial pressure-volume relationship, dynamic compliance and arterial blood pressure waveform.
\end{abstract}

Key words: non-invasive finger blood pressure, dynamic compliance, arterial pressure-volume relationship, pulse waveform, modelling.

\section{INTRODUCTION}

The non-linear response of the arterial wall-to-transmural pressure changes serves as a basis for several non-invasive blood pressure estimation methods. One of them, the oscillometric method, uses the amplitude or the shape of volume oscillations under the cuff area as a criterion for blood pressure estimation $\left.{ }^{1}\right]$. It has been commonly accepted that in most conditions the peak of the cuff plethysmographic oscillations corresponds to the mean intra-arterial blood pressure $\left[{ }^{2-4}\right]$. However, it has also been demonstrated that the maximum amplitude algorithm can be inadequate in some combinations of the arterial wall and pulse parameters $\left[^{5-6}\right]$.

Another method, the volume clamp technique, needs for correct measurement an accurate adjustment of the set-point that should be placed in the area of maximum slope of the S-shaped $P-V$ relationship of the artery (Fig. 1) $\left[^{7-8}\right]$. 

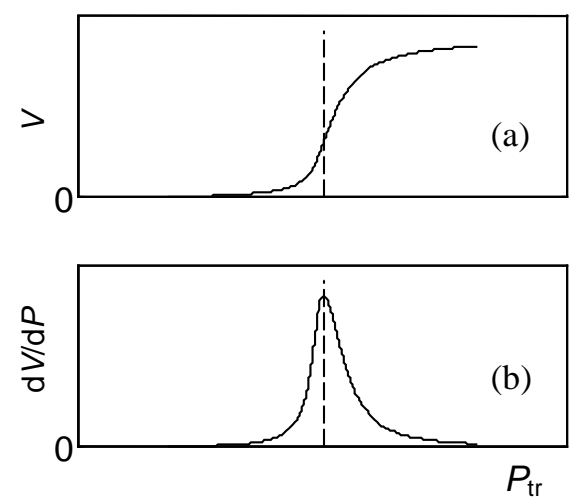

Fig. 1. Arterial pressure-volume $(P-V)$ relationship (a) and its first derivative $\mathrm{d} V / \mathrm{d} P$ (b); $P_{\mathrm{tr}}-$ transmural pressure.

In both above-mentioned methods knowledge of the arterial $P-V$ relationship is of great importance for the correct interpretation of results in indirect blood pressure measurement.

In addition to that, data about patients' arterial $P-V$ relationship or its slope (called "compliance") have an independent prognostic value in the assessment of patients' vascular condition. It has been revealed that an elevated level of arterial blood pressure is usually originated by an increase in the arterial wall stiffness and peripheral vascular resistance. The "compliance" or "elasticity" of an artery has been considered a useful tool in the evaluation of the cardiovascular disease $\left[{ }^{9-10}\right]$. Moreover, the dynamic compliance of arteries, determined in every cardiac cycle and still not frequently used in clinical practice, has a good perspective to become an appreciated measure in future cardiovascular diagnostics $\left[{ }^{11}\right]$.

To determine mechanical properties of peripheral arteries, such as the dynamic vascular compliance, a simultaneous recording of the arterial blood pressure $P_{\mathrm{a}}(t)$ and volumetric signal $V(t)$ are needed. To solve this problem, an invasive technique $\left[{ }^{12}\right]$, the volume clamp method $\left[{ }^{13}\right]$ or tonometry $\left[{ }^{14}\right]$, can be used for blood pressure waveform registration. Volume changes can be recorded by applying impedance plethysmography $\left[{ }^{15}\right]$, photoplethysmography $\left[{ }^{16-17}\right]$, pneumoplethysmography $\left[{ }^{18}\right]$, and ultrasonic $\left[{ }^{19}\right]$ or laser techniques $\left[{ }^{20}\right]$. Response to vibration has also been applied to the analysis of biomechanical characteristics of arteries $\left[{ }^{11}\right]$.

Owing to viscoelastic properties of the arterial wall, the original recordings of $P-V$ characteristics show a hysteresis loop and are different for static and dynamic measurements $\left.{ }^{7,21}\right]$.

The so-called Zener or standard solid model for viscoelastic objects $\left[{ }^{22}\right]$ involves three elements (Fig. 2). At very low frequencies it is possible to measure the static compliance, determined only by the elastic element $E_{2}$. At high frequencies the dynamic compliance is determined by both $E_{1}$ and $E_{2}$.

For pressure changes at or around heart beat frequencies in man $(\sim 1 \mathrm{~Hz})$, one should model the dynamic compliance $\left[{ }^{23}\right]$. Shimazu et al. have also 


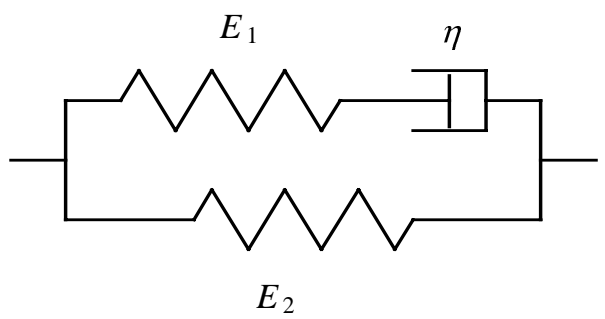

Fig. 2. The Zener model of viscoelasticity; elastic properties are represented by springs $E_{1}$ and $E_{2}$ while creep effects are expressed by dash pot $\eta$.

demonstrated the feasibility of applying the dynamic compliance to the beat-tobeat $P-V$ diagram $\left[{ }^{21}\right]$.

The aim of this study is to estimate the dynamic relationship between the finger volumetric changes and transmural pressure using only photoplethysmographic waveforms recorded at different cuff pressure levels. As a by-product, the finger arterial pressure waveform can also be estimated from the recorded photoplethysmograms and the derived $P-V$ curve.

\section{PRINCIPLES}

Before the choice of suitable methods for modelling the dynamic $P-V$ relationship of the finger arteries, we will survey the method of plethysmography as a valuable source of non-invasive information on volumetric changes in biological tissue. It should be added that in our further analysis the photoplethysmographic signal is treated as an uncalibrated volume signal.

\subsection{Plethysmographic waveform as a function of the transmural pressure}

The transmural pressure $P_{\mathrm{tr}}$ is defined as the difference between the intraarterial pressure $P_{\mathrm{a}}$ and cuff pressure $P_{\mathrm{c}}$ :

$$
P_{\mathrm{tr}}=P_{\mathrm{a}}-P_{\mathrm{c}} .
$$

This formula is valid when the cuff pressure is transmitted to the arteries without attenuation. Introduction of the concept of transmural pressure simplifies the description of the arterial wall condition. Despite the absolute values of intraarterial and cuff pressures, the situation $P_{\mathrm{tr}}=0$ corresponds to the unloaded state of the artery, when internal and external pressures are equal.

The slope of the $P-V$ relationship is known as the vascular compliance $C$ defined as

$$
C=\mathrm{d} V / \mathrm{d} P
$$



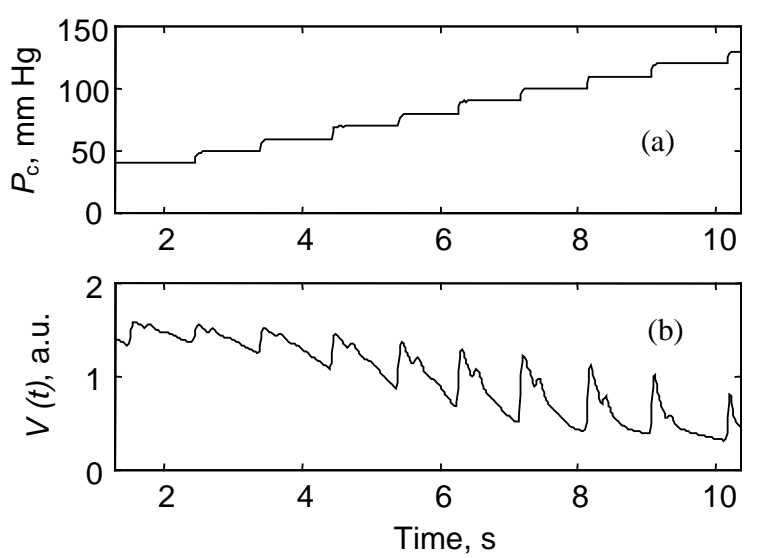

Fig. 3. Simultaneous cuff pressure and photoplethysmographic recordings in the finger; $V(t)$ volumetric (photoplethysmographic) signal.

Figure 3 demonstrates the simultaneous cuff pressure and photoplethysmographic recordings in the finger. The pressure in the cuff is increased by equal steps. Every rise in the pressure is cardio-synchronized allowing the recording of photoplethysmographic pulses at constant cuff pressure levels.

As can be seen in Fig. 3b, the shapes of plethysmographic pulses strongly depend on the cuff pressure (and, subsequently, on the transmural pressure). Arterial pressure changes are transformed into volume changes with a variable gain determined by the altering value of the slope of the $P-V$ relationship.

It can be concluded that the variable waveform of plethysmographic pulses carries information about the $P-V$ relationship and vascular compliance of the tissue and transmural pressure.

We shall demonstrate that it is sufficient to know the plethysmograms of different shapes, recorded at known cuff pressures, to build the $P-V$ diagram and locate the point of maximum compliance. In other words, the $P-V$ curve will be derived without the measurement of the blood pressure $P_{\mathrm{a}}(t)$.

\subsection{Search for the basic algorithm}

We assume that the arterial volumetric signal $V$ depends only on transmural pressure $P_{\text {tr }}$ and that the relationship is non-linear:

$$
V=f\left(P_{\mathrm{tr}}\right)=f\left(P_{\mathrm{a}}-P_{\mathrm{c}}\right) .
$$

There are two unknowns in (3): pressure $P_{\mathrm{a}}$ and function $f\left(P_{\mathrm{tr}}\right)$. Estimation of the input $P_{\mathrm{tr}}$ and $P-V$ model parameters from the output $V$ is possible only owing to a special shape of the $P-V$ relationship of the artery, namely, to the existence of a region with higher compliance between two regions of lower compliance. 
If we have simultaneously recorded two volumetric signals at different cuff pressure levels, the same relationship is valid for both of them:

$$
V_{1}=f\left(P_{\mathrm{tr} 1}\right), \quad V_{2}=f\left(P_{\mathrm{tr} 2}\right),
$$

or

$$
P_{\mathrm{tr} 1}=f^{-1}\left(V_{1}\right), \quad P_{\mathrm{tr} 2}=f^{-1}\left(V_{2}\right) .
$$

According to Eq. (1), the relevant transmural pressures are determined as:

$$
P_{\mathrm{tr} 1}=P_{\mathrm{a}}-P_{\mathrm{c} 1}, \quad P_{\mathrm{tr} 2}=P_{\mathrm{a}}-P_{\mathrm{c} 2} .
$$

In (6) the arterial pressure $P_{\mathrm{a}}$ is common for both equations. When two volumetric signals are not recorded during the same cardiac cycle but in successive cycles, we have to assume that the intra-arterial pressure wave is invariable during these cycles.

By combining (5) and (6), we obtain

$$
f^{-1}\left(V_{1}\right)=P_{\mathrm{a}}-P_{\mathrm{c} 1}, \quad f^{-1}\left(V_{2}\right)=P_{\mathrm{a}}-P_{\mathrm{c} 2} .
$$

Now we can eliminate the unknown input signal $P_{\mathrm{a}}$ by subtracting in (7) the second equation from the first one:

$$
f^{-1}\left(V_{1}\right)-f^{-1}\left(V_{2}\right)=P_{\mathrm{c} 2}-P_{\mathrm{c} 1} .
$$

Equation (8) shows that the difference between the transmural pressures is equal to the difference in cuff pressures. Equation (8) can be used as a basic equation for further $P-V$ modelling. However, the intercept of the function $P_{\mathrm{tr}}=f^{-1}(V)$ cannot be determined by applying this algorithm. To overcome this shortcoming, an additional criterion should be introduced. A reasonable assumption about the vascular compliance, having its maximum value at zero transmural pressure, can serve as an independent criterion in computing the intercept:

$$
f^{-1}(V)=0, \quad \text { if } \quad C=\max .
$$

\subsection{Functions for $S$-shaped $P-V$ curve parameter fitting}

In mathematical studies the arterial wall S-curve is usually modelled by using exponents $\left[{ }^{5-6}\right]$ or the arctangent function $\left[{ }^{24}\right]$. However, experimental recordings of the dynamic vascular compliance of finger arteries, carried out by Peñaz $\left[{ }^{11}\right]$, revealed a different shape of the curve than that expected from the above-given models. Therefore the choice of the function for a successful approximation of the finger arterial $P-V$ relationship remains still an issue to be examined in further studies. 
It should be pointed out that our basic equation (8) contains the inverse form of the $P-V$ relationship and that actually we seek the $V-P$ relationship $\left(P_{\mathrm{tr}}=f^{-1}(V)\right)$. A number of functions, which can potentially be used in arterial $P-V$ model parameter fitting, are listed in Table 1.

Table 1. A list of functions suitable for modelling the relationship $P_{\mathrm{tr}}=f^{-1}(V)$

\begin{tabular}{l|c|c}
\hline \multicolumn{1}{c|}{ Model } & Abbr. & \multicolumn{1}{c}{ Formula } \\
\hline Polynomial & $\mathrm{P}$ & $P_{\mathrm{tr}}=\sum_{k=0}^{n} A_{k} V^{k}$ \\
$\begin{array}{lcl}\text { A combination of Chebyshev } \\
\text { polynomials }\end{array}$ & $\mathrm{CP}$ & $P_{\mathrm{tr}}=\sum_{k=0}^{n} A_{k} T_{k}(V)$, \\
& where $T_{0}(V) \ldots T_{n}(V)$ are Chebyshev polynomials \\
Tangent & $\mathrm{T}$ & $P_{\mathrm{tr}}=\frac{1}{A_{2}} \tan \left(\frac{V-V_{0}}{A_{1}}\right)$ \\
& $\mathrm{AT}$ & $P_{\mathrm{tr}}=\frac{A_{1}}{A_{3}} \tan \left(\frac{V-V_{0}}{A_{1}}\right)$ for $V-V_{0} \geq 0$ \\
& & $P_{\mathrm{tr}}=\frac{A_{2}}{A_{3}} \tan \left(\frac{V-V_{0}}{A_{2}}\right)$ for $V-V_{0}<0$
\end{tabular}

Asymmetric natural logarithm

$$
\begin{aligned}
P_{\mathrm{tr}} & =-\frac{A_{1}}{A_{3}} \ln \left(1-\frac{V-V_{0}}{A_{1}}\right) \text { for } V-V_{0} \geq 0 \\
P_{\mathrm{tr}} & =\frac{A_{2}}{A_{3}} \ln \left(1+\frac{V-V_{0}}{A_{2}}\right) \text { for } V-V_{0}<0
\end{aligned}
$$

Polynomial models $\mathrm{P}$ and $\mathrm{CP}$ can well fit experimental data. However, their drawback is that the solution is not always meaningful from the physiological point of view. It means, for instance, that the compliance curve (Fig. 1) may have several local maximum points when a high-order polynomial is applied, and it fits poorly experimental data when a low-order polynomial is used.

$V-V_{0}$ is the arterial volume corresponding to zero transmural pressure in models T, AT and AL.

When applying symmetrical model $\mathrm{T}$, it is assumed that the sensitivity of the model is the same for positive and negative transmural pressures. However, this assumption does not hold in practice.

Asymmetric models AT and AL are free from the limitations concerning symmetric models. It should be noted that the compliance curve for the AL model has a sharp maximum at $P_{\mathrm{tr}}=0$. This feature is obviously inadequate 
from the physiological point of view: switching between positive and negative transmural pressures is not so sharply expressed in reality. To model the region of near-zero transmural pressure independently from the other parts of the curve, an additional parameter should be used.

From the computational point of view the polynomial models can be obtained by solving linear overdetermined systems of equations, whereas models T, AT and AL need an iterative solution. In case of iteration, an increase in the number of parameters causes a decrease in robustness as well as in computation speed.

In our previous study $\left.{ }^{6}\right]$ we applied an asymmetric exponential model for the $P-V$ relationship $V=f\left(P_{\text {tr }}\right)$, in which one exponent was used for positive and another for negative pressures. In the present model, to obtain the inverse form of the relationship $P_{\mathrm{tr}}=f^{-1}(V)$, we decided to use preferably the asymmetric tangent approximation.

\section{METHODS}

The volunteer, a healthy 25 year old male was studied at rest at room temperature $22-23^{\circ} \mathrm{C}$. The subject was seated comfortably with the left arm resting at heart level.

Photoplethysmograms were recorded by applying one channel of the UT9201 physiograph (University of Tartu, Estonia) containing a transmittance mode photoplethysmograph. The finger cuff with a light emitter and photodetector was wrapped around the middle phalanx of the middle finger of the subject's left hand. The pressure in the cuff was increased by equal steps of $10 \mathrm{~mm} \mathrm{Hg}$. Every rise in the pressure was cardio-synchronized by allowing recording of the photoplethysmographic pulses at constant cuff pressure levels. Diastolic parts of two successive photoelectric pulses with relatively large amplitude and remarkably different shapes were selected for further analysis.

For modelling the finger arterial $P-V$ relationship we used an asymmetric tangent approximation (Table 1).

The Levenberg-Marquardt method was used to identify model parameters $A_{1}, A_{2}, A_{3}$ and $V_{0}$ according to Eq. (8).

\section{RESULTS}

An example of applying the described method to estimate the finger arterial pressure-volume relationship and blood pressure waveform from two successive photoplethysmographic pulses is shown in Fig. 4.

Parts a, b and c in Fig. 4 are interrelated, transmural pressures (part c) are related to volumetric curves (part a) through a non-linear $P-V$ relationship (part b), obtained by the non-linear fitting by applying an asymmetric tangent approximation. 


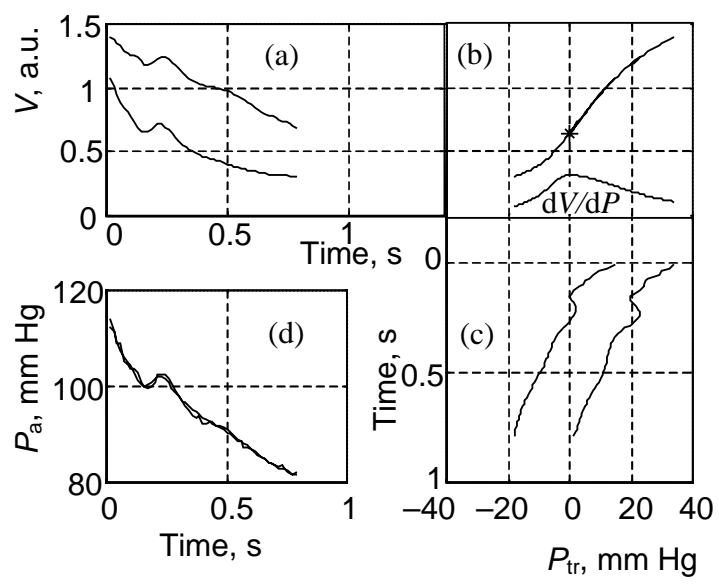

Fig. 4. Experimentally recorded diastolic parts of two photoplethysmographic pulses (a), estimated $P-V$ relationship (b), calculated transmural pressures (c) and arterial pressure waveforms (d).

It can be noticed that the difference between transmural pressure pulses (Fig. 4c) is approximately constant and equal to the difference between cuff pressure values. The cuff pressure was held at 80 and $100 \mathrm{~mm} \mathrm{Hg}$ during the recording of these two pulses.

The waveforms of arterial pressure (Fig. 4d) are similar, demonstrating reliability of the applied model.

\section{DISCUSSION}

In our analysis we have made several assumptions and simplifications.

1. We have assumed that the volumetric signal is a function of a single variable, the transmural pressure. It means that the cuff pressure is transmitted to the arterial wall without attenuation. This assumption is used in most of the methods of non-invasive blood pressure estimation and is generally considered reasonable $\left[{ }^{5}\right]$.

2. An assumption about the vascular compliance having its maximum value at zero transmural pressure was made. This assumption is also commonly accepted $\left[{ }^{5}\right]$.

3. Since we have assumed that the transmural pressure and volume are related only through the dynamic $P-V$ relationship, there are no phase shifts between the transmural pressure and volume during the diastolic part of a cardiac cycle. In reality the computed pressure waveform can be slightly different from the true arterial pressure waveform, especially in the (near-) systolic part of the pulse wave, due to the time-dependent component in the real relationship between the pressure and volume.

4. We have assumed that there are no creep effects during the cardiac cycle. 
As mentioned in Introduction, it is reasonable to take into consideration only the "dynamic compliance" when pressure changes at the frequency of about $1 \mathrm{~Hz}$ are studied $\left[{ }^{23}\right]$. In fact, however, viscous effects can cause some low frequency drifts in the volumetric signal. This volumetric component of the signal, if present, should be subtracted before applying the computing algorithm. As a rule, the removal of viscous drift components is a complicated task. It can be partly solved by correcting the DC level of the signals taking into account the behaviour of the AC components.

There are some assumptions, which are essential only in case of using successively recorded volumetric pulses, but are not necessary if volumetric pulses are recorded simultaneously at different cuff pressures at different sites (e.g., in adjacent fingers). In this case the arterial pressure is truly the same for both pulses. A disadvantage of this approach is that a two-channel photoplethysmographic device is needed and channels need to be calibrated to get the same sensitivity to transmural pressure changes.

In the present study, the one-channel recording of successive pulses instead of the two-channel parallel recording was chosen only for the sake of simplicity and clarity of introducing the new method. It introduces two additional conditions: 1) the intra-arterial pressure wave is invariant during the period of successive pulses and 2) the arterial $P-V$ relationship is invariant during the period of successive pulses.

It should also be emphasized that some restrictions, applied to the experimental set-up, were not necessary from the viewpoint of the proposed algorithm. An invariant level of the counter pressure during every cardiac cycle (Fig. 3) and the constant difference between the counter pressure levels of two selected pulses are used only for the sake of clarity.

The two plethysmographic pulses should have markedly different shapes; otherwise there is no sufficient information for the reconstruction. On the other hand, the plethysmograms should partly overlap in the central region of the Scurve. To increase the amount of information, more than two successive cardiac cycles can be involved. In this case, Eq. (8) should be used in an expanded form

$$
f^{-1}\left(V_{1}\right)-f^{-1}\left(V_{m}\right)=P_{\mathrm{c} m}-P_{\mathrm{c} 1},
$$

where $m$ can be $2,3, \ldots$

\section{CONCLUSION}

The results of modelling demonstrated that two finger photoplethysmographic waveforms, recorded at different cuff pressure levels, can be used for the estimation of the finger arterial pressure-volume relationship, dynamic compliance and arterial blood pressure waveform. 


\section{ACKNOWLEDGEMENT}

This research was supported by the Estonian Science Foundation (grant No. 5280).

\section{REFERENCES}

1. Marey, E. J. La méthode graphique dans les sciences expérimentales et principalement en physiologie et en médecine. Masson, Paris, 1878.

2. Posey, J. A., Geddes, L. A., Williams, H., and Moore, A. G. The meaning of the point of maximum oscillations in cuff pressure in the indirect measurement of blood pressure, Part I. Cardiovasc. Res. Cent. Bull., 1969, 8, 15-25.

3. Mauck, G. W., Smith, C. R., Geddes, L. A., and Bourland, J. D. The meaning of the point of maximum oscillations in cuff pressure in the indirect measurement of blood pressure, Part II. J. Biomech. Eng., 1980, 102, 28-33.

4. Sapinski, A. Theoretical basis for proposed standard algorithm of blood pressure measurement by the sphygmooscillographic method. J. Clin. Eng., 1997, 22, 171-174.

5. Baker, P. D., Westenskow, D. R., and Kück, K. Theoretical analysis of non-invasive oscillometric maximum amplitude algorithm for estimating mean blood pressure. Med. Biol. Eng. Comput., 1997, 35, 271-278.

6. Raamat, R., Talts, J., Jagomägi, K., and Länsimies, E. Mathematical modelling of non-invasive oscillometric finger mean blood pressure measurement by maximum oscillation criterion. Med. Biol. Eng. Comput., 1999, 37, 784-788.

7. Wesseling, K. H., de Wit, B., van der Hoeven, G. M. A., van Godoever, J., and Settels, J. J. Physiocal, calibrating finger vascular physiology for Finapres. Homeostasis, 1995, 36, 67-82.

8. Peñaz, J. Criteria for set point estimation in the volume clamp method of blood pressure measurement. Physiol. Res., 1992, 41, 5-10.

9. Cohn, J. N. Vascular wall function as a risk marker for cardiovascular disease. J. Hypertens., 1999, 17, Suppl. 5, 41-44.

10. Da Silva, M. P. Arterial compliance: a tool in the evaluation of vascular disease in familial hypercholesterolemia. Atherosclerosis, 2000, 148, 437-440.

11. Peñaz, J., Honzikova, N., and Jurak, P. Vibration plethysmography: a method for studying the visco-elastic properties of finger arteries. Med. Biol. Eng. Comput., 1997, 35, 633-637.

12. Gow, B. S. Circulatory correlates: vascular impedance, resistance and capacity. In Handbook of Physiology, Sec. 2, Vol. 2. The Cardiovascular System (Bohr, D. F., Somlyo, A. P., and Sparks, H. V., Jr., eds.), American Physiol. Soc., Bethesda, 1980, 353-408.

13. Gizdulich, P. and Wesseling, K. H. Forearm arterial pressure-volume relationships in man. Clin. Phys. Physiol. Meas., 1988, 9, 123-132.

14. Lenard, Z., Fulop, D., Visontai, Z., Jokkel, G., Reneman, R., and Kollai, M. Static versus dynamic distensibility of the carotid artery in humans. J. Vasc. Res., 2000, 37, 103-111.

15. Kawarada, A., Shimazu, H., Ito, H., and Yamakoshi, K. Noninvasive measurement of arterial elasticity in various human limbs. Med. Biol. Eng. Comput., 1988, 26, 641-646.

16. Shelley, K. H., Murray, W. B., and Chang, D. Arterial-pulse oximetry loops: a new method of monitoring vascular tone. J. Clin. Monit., 1997, 13, 223-228.

17. Lopez-Beltran, E. A., Blackshear, P. L., Finkelstein, S. M., and Cohn, J. N. Non-invasive studies of peripheral vascular compliance using a non-occluding photoplethysmographic method. Med. Biol. Eng. Comput., 1998, 36, 748-753.

18. Brinton, T. J., Cotter, B., Kailasam, M. T., Brown, D. L., Chio, S. S., O'Connor, D. T., and DeMaria, A. N. Development and validation of a noninvasive method to determine arterial pressure and vascular compliance. Am. J. Cardiol., 1997, 80, 323-330. 
19. Brands, P. J., Hoeks, A. P., Willigers, J., Willekes, C., and Reneman, R. S. An integrated system for the non-invasive assessment of vessel wall and hemodynamic properties of large arteries by means of ultrasound. Eur. J. Ultrasound, 1999, 9, 257-266.

20. Meigas, K., Hinrikus, H., Kattai, R., and Lass, J. Self-mixing in a diode as a method for cardiovascular diagnostics. J. Biomed. Opt., 2003, 8, 152-160.

21. Shimazu, H., Fukuoka, M., Ito, H., and Yamakoshi, K. Noninvasive measurement of beat-tobeat vascular viscoelastic properties in human fingers and forearms. Med. Biol. Eng. Comput., 1985, 23, 43-47.

22. Schiessel, H., Metzler, R., Blumen, A., and Nonnemacher, T. F. Generalized viscoelastic models: their fractional equations with solutions. J. Phys. A: Math. Gen., 1995, 28, 65676584.

23. Wesseling, K. Physics of the cardiovascular system. In Proc. International School of Physics. Varenna, 1979. North-Holland, Amsterdam, 1981, 66-161.

24. Wesseling, K. H., Jansen, J. R. C., Settels, J. J., and Shreuder, J. J. Computation of aortic flow from pressure in humans using a nonlinear, three element model. J. Appl.Physiol., 1993, 74, $2566-2573$.

\title{
Sõrme arteriaalse rõhu-mahu sõltuvuse ja vererõhulaine kuju määramine fotopletüsmograafiliste signaalide alusel
}

\author{
Jaak Talts
}

On esitatud meetod sõrme arterite rõhu-mahu $(P-V)$ sõltuvuse ja arteriaalse vererõhukõvera määramiseks kahe erineval mansetirõhul registreeritud fotopletüsmograafilise (volumeetrilise) signaali põhjal. Meetod võimaldab määrata ka väikeste arterite dünaamilist venitatavust iga südametsükli keskmisena ja venitatavuse hetkväärtusi. Arteriaalse $P-V$ kõvera modelleerimisel kasutati asümmeetrilist tangenslähendust. Modelleerimise tulemused kinnitavad kirjeldatud meetodi praktilist kasutatavust. 